\title{
Educación en Arquitectura: Transiciones hacia el Desarrollo Sostenible
}

\author{
Education in Architecture: Transitions towards Sustainable Development
}

Educação em Arquitetura: Transições para o Desenvolvimento Sustentável

\author{
Alexandra Isabel Castellanos Tuirán. \\ Arquitecta, M.Sc Desarrollo y ambiente \\ Corporación Universitaria del Caribe-CECAR, Sincelejo. \\ alexandra.castellanos@cecar.edu.co \\ (iD) https://orcid.org/0000-0003-3423-8714 \\ Pedro Arturo Martínez Osorio \\ Arquitecto, Ph.D. Design. \\ Corporación Universitaria del Caribe-CECAR, Sincelejo. \\ pedro.martinez@cecar.edu.co \\ (iD) https://orcid.org/0000-0002-9024-0918
}

Recibido: enero 5 de 2020

Aceptado: febrero 10 de 2020

Publicado: febrero 15 de 2020

\section{RESUMEN}

Se analizan tres aspectos relativos a la Educación en Arquitectura, con el fin de encontrar puntos en común y convergencias: el desarrollo social de la cultura occidental, desde el punto de vista ambiental; la evolución de la Educación en occidente, desde la aparición de las primeras teorías modernas; y el proceso evolutivo de la formación en Arquitectura desde la perspectiva histórica y epistemológica. Se reflexiona sobre la relación de estos temas, desde una visión crítica de la enseñanza de la Arquitectura, una disciplina en la que muchas veces ha de pensarse que estos aspectos transitan por caminos divergentes.

Palabras clave: Ambiente; Educación en Arquitectura; Desarrollo Sostenible

\section{ABSTRACT}

Three aspects related to Education in Architecture are analyzed in order to find common ground and convergences: the social development of Western culture, from the environmental point of view; the evolution of Education in the West, since the appearance of the first modern theories; and the evolutionary process of Architecture training from a historical and epistemological perspective. It reflects on the relationship of these issues, from a critical view of the teaching of Architecture, a discipline in which many times it must be thought that these aspects travel along divergent paths.

Keywords: Environment; Education in Architecture; Sustainable Development 


\section{RESUMO}

Três aspectos relacionados à Educação em Arquitetura são analisados para encontrar bases comuns e convergências: o desenvolvimento social da cultura ocidental, do ponto de vista ambiental; a evolução da Educação no Ocidente, desde o surgimento das primeiras teorias modernas; e o processo evolutivo da formação em Arquitetura de uma perspectiva histórica e epistemológica. Reflete sobre a relação dessas questões, a partir de uma visão crítica do ensino da Arquitetura, disciplina em que muitas vezes se deve pensar que esses aspectos percorrem caminhos divergentes.

Palavras-chave: Meio Ambiente; Educação em Arquitetura; Desenvolvimento Sustentável

\section{INTRODUCCIÓN}

Se analizan en este artículo tres aspectos de gran importancia que están relacionados con el tema de la Educación en Arquitectura: Desarrollo-Ambiente, Educación-Cultura, y Formación Disciplinar. La idea es destacar algunos aspectos fundamentales en la evolución de esos conceptos, para, finalmente, contrastar y encontrar convergencias y desencuentros entre los elementos de análisis.

El documento se ha dividido en cuatro partes. En la primera, se realiza un recuento muy sucinto de algunos aspectos significativos en la evolución de la idea de desarrollo social, desde un enfoque centrado en la problemática ambiental. Se exponen algunos de los acontecimientos trascendentales en la discusión sobre la problemática entre Desarrollo y Ambiente, con el fin de explicar el momento coyuntural que sobrevivimos en la actualidad.

En la segunda parte, se exponen algunos aspectos fundamentales del panorama de la Educación, poniendo especial atención a los problemas y retos que plantea el mundo contemporáneo para la Educación de Futuro.

La tercera parte, hace un esbozo muy sucinto de aspectos claves sobre el desarrollo de la Educación en Arquitectura a través de la historia, para, en la cuarta parte, generar un cruce de caminos, contrastes y convergencias entre los diferentes elementos de análisis.

\section{Desarrollo, Sociedad y Territorio: Una Visión desde la Problemática Ambiental}

Partiendo de la idea del desarrollo en la historia social de la cultura occidental, desde una visión enfocada en la producción, la economía y cómo esta afecta la apropiación del hombre sobre el mundo y su entorno, se puede encontrar un punto trascendental de cambio actitudinal sobre la tendencia ancestral de relación entre humanos, ambiente y producción, en el conjunto de hechos que dieron paso en la segunda mitad del siglo XVIII a la Revolución de la Industrialización que se dio inicio en Inglaterra, en donde se cambia la forma de apropiación del territorio basada en la autosuficiencia de las localidades, por un escenario donde se potencializa la industrialización de los procesos y la división social, tanto del trabajo como de la producción, desarrollando un nuevo tipo de sociedad industrializada, diferenciada del esquema tradicional de sociedad que la había precedido (Ballesteros y Pérez, 1997).

La idea de la masificación de la producción generó grandes cambios, tanto en la estructura de los grupos sociales como en la forma de apropiación del territorio. Tanto así, que ese modelo de relación, Sociedad-Medio-Producción, evolucionó a lo largo de todo el siglo XIX, trasciende con la expansión del modelo capitalista y desemboca en la segunda mitad del siglo XX en un pensamiento que pugna por una globalización de la economía (hablando en términos de mercado), lo cual se convierte en una institución que reglamenta la convivencia universal (Leff, 2000), así como los valores y símbolos culturales globales, mediados por una expansión y revolución digital del conocimiento, que es promocionado a través los medios de comunicación.

La Internet y los sistemas de comunicación por telefonía móvil, las diferentes redes sociales, han cambiado el panorama y la forma de desarrollar las actividades sociales del hombre en casi todos los rincones de planeta; la velocidad de interacción y respuesta condicionan la forma de vida y las nuevas necesidades de los seres humanos. 
En términos económicos, se pasa de una sociedad de intercambio a una sociedad de consumo, en la que poco importan las consecuencias ambientales de esta forma de interacción social, siendo esto una manifestación del desigual reparto, tanto de la riqueza como de las maneras de apropiar y utilizar los recursos naturales. Un claro ejemplo de esta problemática, se refleja en un país como Estados Unidos, con solo el 4,5\% de la población mundial, en el cual se producen aproximadamente el $33 \%$ de todos los desechos sólidos del mundo (Ballesteros y Pérez, 1997).

En los últimos 50 años este modelo de apropiación del territorio, enfocado en la producción y el consumo, se ha vuelto insostenible. Fuertes cambios en los ecosistemas producto de la apropiación y explotación no sostenible de los recursos naturales, han generado una crisis ambiental que no es ajena a las realidades de los grupos humanos.

Esta crisis producto de la globalización de los procesos de explotación del medio, desde la visión del consumo y la acumulación del capital, ha desencadenado una serie de discusiones teóricas al respecto de la relación Humano-AmbienteTerritorio, lo cual ha trascendido los ámbitos académicos y ha generado una conciencia de la problemática ambiental que ha producido tanto una postura crítica al respecto, como lo que se puede considerar su contraparte, la llamada "revolución verde" (FAO, 1996).

Es así como la problemática ambiental ocupa en la actualidad un lugar trascendental en las discusiones sobre desarrollo sostenible de la humanidad. Una de las primeras manifestaciones mundiales alrededor de este tema se encuentra en la Declaración de la Conferencia de las Naciones Unidas sobre el Medio Ambiente Humano, celebrada en Estocolmo en 1972. En ella se atiende la problemática de la degradación ambiental y la contaminación transfronteriza, proclamando, entre otros aspectos, que los elementos naturales y artificiales del medio ambiente humano son esenciales para el bienestar del hombre, y que el medio es el que le da el sustento material y le brinda la oportunidad de desarrollarse; esto como un primer paso en la construcción de pensamiento y conciencia de la problemática a escala internacional.

En la llamada "Cumbre de la Tierra" la conferencia de las naciones Unidas sobre el medio ambiente y el desarrollo, llevada a cabo en Río de Janeiro, Brasil, en
1992, se reconoce internacionalmente el hecho de que la protección del ambiente y la administración de recursos naturales, necesitan integrarse a los aspectos socio-económicos, surgiendo la nueva idea, a nivel internacional, de que los problemas del ambiente en el planeta Tierra, están íntimamente relacionados con las condiciones económicas, con la justicia y la equidad social.

Para el año 2009, se lleva a cabo la Tercera Conferencia Mundial sobre Cambio Climático en Copenhague, que pretendía establecer compromisos para la reducción de las emisiones de $\mathrm{CO}^{2}$ en al menos un $50 \%$ en 2050 respecto a 1990. Sin embargo, el acuerdo no fue aprobado por todos los países, y las conversaciones no lograron los compromisos esperados. La conferencia se realiza anualmente, y en el 2019 se llevó a cabo su versión 25 , en la cual se mantiene el deseo de alcanzar disminuciones de emisiones de $\mathrm{CO}^{2}$ al menos en un $45 \%$ para 2030. (Climate Action Summit, 2019).

En atención a esas mismas problemáticas, en 2015, 193 países adoptaron como parte de una nueva Agenda de Desarrollo mundial, una serie de objetivos para erradicar la pobreza, proteger el planeta y garantizar la prosperidad para todos. Esos objetivos fueron Ilamados Objetivos de Desarrollo Sostenible (ODS), y tienen un conjunto de 169 metas específicas que se espera sean logradas en los próximos 15 años (DNP, 2019).

Como parte de esta iniciativa, en Colombia se estableció la Agenda 2030, que se convierte en una hoja de ruta para el desarrollo sostenible en el país, la cual propone una serie de metas que requieren la ejecución de acciones con una visión a largo plazo, que se convierten en una apuesta por un enfoque de desarrollo que incluye, además de la dimensión económica, la dimensión social, ambiental y de prosperidad. En este sentido, los programas y políticas que se desarrollen en el país en los próximos años buscarán ser cada vez más enfáticos y firmes con el logro de esos propósitos (DNP, 2019).

Entre los 17 ODS para erradicar la pobreza y garantizar la equidad y protección del planeta, se destaca el Objetivo 11, denominado "Ciudades y Comunidades Sostenibles", cuya intención es lograr ciudades y asentamientos humanos inclusivos, seguros, resilientes y sostenibles. Entre las metas hacen parte del Objetivo 11, se encuentran: la protección del patrimonio cultural y natural; 
minimizar el impacto ambiental de las ciudades; apoyo en la construcción sostenible y resiliente; entre muchas otras.

También en ese escenario de fuertes tensiones, cada vez surgen con mayor fuerza indicios de la conformación de una conciencia del desarrollo social, desde una visión del desarrollo sostenible, y la apropiación más armónica del territorio. Prueba de ello, son las numerosas organizaciones no gubernamentales en pro de la protección del ambiente y los recursos naturales, bióticos, animales y humanos. Así también la creciente popularización del llamado consumo ecológico, desde el cual el consumidor empieza a ser consciente de su papel fundamental en el cambio, y de su vital contribución para frenar el deterioro del medio natural (Ballesteros y Pérez, 1997).

Ante estas opciones, surgen posiciones contradictorias, que establecen una discusión en torno a la problemática del consumo ecológico como solución al problema ambiental, el cual, desde esta interpretación, no puede constituirse por sí mismo en una solución, debido a que este presupone el mismo grado de consumo, solo que incorporando la variable ecológica en la elección del producto.

En sintonía con esas reflexiones, algunas formas de organización social se pueden identificar en diferentes contextos, las cuales intentan responder de mejor forma a los retos producto de la crisis social y ecológica. Estas iniciativas locales son conocidas como Redes para la Transición (Sistig, 2018), con un liderazgo y visibilidad que se puede rastrear hacia 2006 en Totnes (Inglaterra), pero que hoy en día se pueden identificar movimientos sociales similares en más de 50 países (Red de Transición, 2020).

Lo más importante para esas iniciativas locales de transición es la concientización a la comunidad sobre la necesidad de construir un nuevo modelo económico y social (Red de Transición, 2020). Entre los conceptos que sobresalen se pueden identificar: resiliencia comunitaria, autosuficiencia, rechazo del sistema convencional de producción y consumo, recuperación del intercambio, la cooperación, colaboración, solidaridad y el bien común, decrecimiento, ecologismo, feminismo y democracia (Red de transición, 2020).
En la actualidad el planeta sobrevive una pandemia por causa del virus SARS-CoV-2, mundialmente conocido como COVID-19. Esta situación de crisis genera muchos interrogantes al respecto de lo positivo o negativo que puede llegar a ser para el planeta. Es innegable que se han generado implicaciones de forma directa o indirecta sobre el ambiente, como por ejemplo el mejoramiento de la calidad del aire en algunas de las ciudades con mayor polución (Somani, Srivastava, Kumar, y Sharma, 2020; Lochandwala y Gautam, 2020; Madurai y Pugazhendhi, 2020).

Madurai y Pugazhendhi (2020), hablan sobre la rápida adaptación tecnológica que se ha tenido durante la pandemia, y su influencia sobre la sociedad y el ambiente. Para los autores, durante los últimos meses se ha desarrollado una re estructuración social y del ambiente para el control de la pandemia. Cambios en las tecnologías y protocolos de atención en salud; cambios en los procesos de dirección y toma de decisiones por parte de los gobiernos y administraciones locales; adaptación en los niveles de privacidad de los individuos en orden de garantizar la salud de los grupos humanos; cambios en los sistemas de cooperación internacional; así como las relaciones a nivel público y privado, entre otros, son algunos de los ajustes que se están generando como forma de adaptación a una situación extremamente compleja que seguramente transformará radicalmente la forma de vivir en el planeta.

Es así que este panorama permite entrever que, a comienzos de la segunda década del siglo XXI, además de la situación de pandemia mundial por la COVID-19, se sobrevive un momento coyuntural, acelerado por la situación de emergencia, una crisis global, producto de una forma de apropiación inequitativa de los recursos, que ha generado daños difíciles de revertir; y la preocupación cada vez mayor que se tiene como especie comprometida con su desarrollo y el de las futuras generaciones, por lograr otras posibilidades como parte de un ecosistema integral.

\section{Educación y Cultura: Los Problemas Contemporáneos}

El cambio conceptual de la visión tradicionalista de la Educación es un pensamiento que está todavía en vías de construcción; comienza desde las iniciativas de las primeras teorías modernas sobre Educación en el siglo XVIII, con Rousseau 
y el lluminismo, desde los cuales trasciende y evoluciona desde diferentes aportes y visiones de pensadores como Pestalozzi, Herbart, Dewey, entre otros.

Los cambios más significativos en torno a este proceso de evolución del pensamiento pedagógico, se pueden identificar desde las intenciones de desvanecer los límites del aula y la escuela, en una visión que ha generado procesos tan revolucionarios como la Educación mediada por las Tecnologías de la Comunicación, siendo una de sus más antiguas manifestaciones, la Educación por Correspondencia, y la Educación A Distancia, a través de la radio o la televisión.

Desde la década de los sesenta y principalmente con el boom del uso de los computadores en los ochenta, el desarrollo tecnológico ha influenciado fuertemente todos los ámbitos en la vida de los grupos humanos. A nivel educativo, el avance en Ambientes Virtuales de la Educación y la Internet, han generado recientemente, un cambio en la forma de acercarse los jóvenes al conocimiento, con apoyo de un sistema integrado que representa una inagotable fuente de recursos desde los cuales interactuar en la búsqueda del conocimiento.

Uno de los cambios trascendentales en la manera de abordar el compromiso del aprendizaje por parte de Alumno, radica en la posibilidad de autogestionar el conocimiento, a través de la mediación de recursos y ambientes virtuales que facilitan la no presencialidad de los procesos, trayendo esto consigo, poco a poco, la Virtualización de los Procesos Educativos; en este proceso el Docente, quien en el sistema tradicional era el centro y actor principal en el proceso, pasa ahora a un segundo plano, cumpliendo un papel de mediador y facilitador del encuentro del Alumno con el conocimiento.

Las nuevas tendencias, en cuanto al espacio educativo, han empezado a entender esta problemática, y a plantear soluciones viables. En este sentido, son evidentes los esfuerzos desarrollados por sistemas como Google Classroom, Moodle, Blackboard, Microsoft Teams, etc., a través de los cuales es posible gestionar cursos en línea mediante plataformas LMS (Learning Management System) y la gran cantidad de ofertas para el desarrollo de Aprendizaje A Distancia. O, desde la perspectiva pedagógica, las diferentes teorías que hablan de la virtualización y la desescolarización de la Educación.
La situación de pandemia mundial aceleró la rápida adopción de gran parte de esos sistemas y medios educativos, con la finalidad de brindar una seguridad en salud a Estudiantes y Docentes, así también para ofrecer opciones virtuales robustas, sencillas y accesibles (Wijesooriya, Mishra, Brand, y Rubin, 2020).

livari, Sharma, y Venta-Olkkonen (2020), explican la transformación hacia ámbitos digitales en muchos aspectos de la vida diaria durante la pandemia producto de la COVID-19. Los autores afirman que el sistema educativo actual se ha forzado para encajar ante las necesidades de aislamiento, pero que, a nivel mundial, aún prevalecen brechas en el acceso a la tecnología que influyen en las capacidades tecnológicas, sobre todo en la Educación Básica, pero que afectan a todos los niveles educativos.

Las necesidades actuales del sistema social, exigen a la Educación, una postura crítica y flexible, de visión interdisciplinaria, que atienda tanto las necesidades cambiantes y dinámicas de una sociedad globalizada e informatizada, así como el hecho de generar una reflexión al interior sobre la diferencia estructural entre los conceptos de formación integral y formación cognitiva, educar y enseñar. El concepto de formación integral se constituye en una búsqueda de solución a la problemática de la crisis del ser, la cual no es en la actualidad un problema exclusivo de la globalidad, sino que trasciende las acciones y comportamientos locales de los diferentes grupos humanos.

Un estudio muy importante que trabaja en ese sentido, es el denominado Proyecto TuningAmérica Latina 2004-2006, que nace como parte de una búsqueda por fortalecer las estructuras educativas en América Latina y que logra crear un importante escenario de reflexión sobre la Educación Superior y los retos que esta se plantea para responder a las nuevas realidades sociales, económicas y ambientales de la región. Las 4 líneas que se estudian en los Programas Curriculares universitarios son: las competencias genéricas y específicas, los créditos académicos y los enfoques de enseñanza.

Los resultados de ese proyecto permitieron dar a conocer aspectos comunes en la enseñanza y las particularidades de la misma en cada país. A pesar de ser desarrollado con un enfoque de competencias, este ejercicio fue importante en la medida en que permitió a las diferentes instituciones educativas 
de América Latina retroalimentar sus procesos y al mismo tiempo fortalecer redes universitarias con entidades e instituciones público-privadas, garantizando la convergencia de disciplinas y buscando la creación de lo que se consideró como un espacio común de enseñanza superior (Tuning América Latina, 2007).

Por otra parte, con relación a la Agenda de Desarrollo Mundial, promovida por la ONU, a través de los ODS, en el ámbito de la Educación superior, son muchos los aspectos en los que puede contribuir la Universidad al cumplimiento de esos objetivos. Entre las áreas más importantes en las que aporta la Universidad en el logro de los ODS se encuentran: Aprendizaje-Enseñanza, integrando en sus procesos los temas fundamentales para la Educación para la Sostenibilidad (EDS); Investigación, aprovechando las capacidades de Investigación, Innovación y Emprendimiento de los grupos de Investigación, para el desarrollo de proyectos, productos y empresas con enfoque sostenible; gobernanza institucional, políticas de gestión y extensión universitaria, con la cual generar una interrelación mas efectiva de la Universidad con su contexto, enmarcada en términos de la sostenibilidad; liderazgo social, para dinamizar el diálogo y las acciones intersectoriales con una visión sostenible de región y país.

A nivel de competencias, también es importante destacar algunas competencias claves de EDS, con las cuales la Universidad hace efectivo su compromiso de formación con el logro de los ODS, entre ellas: competencia de pensamiento sistémico, para reconocer y analizar sistemas complejos; competencia de anticipación, para comprender y evaluar posibles escenarios futuros; competencia normativa, para reflexionar sobre las normas y valores que subyacen en las acciones de los individuos y grupos; competencia estratégica, para implementar de forma innovadora acciones que fomenten la sostenibilidad; competencia de colaboración, para formas personas sensibles capaces de tener empatía y valorar la diversidad en la resolución de problemas en el marco de la sostenibilidad; competencia de pensamiento crítico, como la capacidad para cuestionar desde una perspectiva histórica, así adoptar una postura que favorezca la toma de decisiones hacia la sostenibilidad; y, finalmente, la competencia de autoconciencia, para comprender el papel de cada uno en la comunidad, la sociedad, como parte de un mismo ecosistema integrado.
De esta forma, a nivel educativo, el panorama contemporáneo presenta algunos aspectos que se destacan: por una parte, las brechas tecnológicas que influyen en gran medida al logro de unos procesos educativos pertinentes para las necesidades y problemáticas actuales; la rápida adopción de sistemas y medios educativos, como estrategia para posibilitar el desarrollo de los procesos académicos en el aislamiento producto de la pandemia, lo cual se ha generado más por necesidad que por un proceso natural de evolución consciente; también, la necesidad urgente de generar una nueva conciencia hacia la sostenibilidad, en la cual el sistema educativo, y ,sobre todo la Universidad, juega un papel fundamental en el logro de las metas y objetivos para hacer posible otros futuros con mejores condiciones para humanos y no humanos.

\section{Arquitectura, Arte y Ciencia: Desarrollo de la Idea de Formación Disciplinar}

Los primeros tratados que marcan una pauta para definir la disciplina de la Arquitectura, y a su vez los procesos que estructuran la formación en esta disciplina, cobran auge desde comienzos del siglo $X V$, aunque ya desde el año 23 A.C "Los diez libros de Arquitectura" de Vitruvio, se destaca como uno de los tratados más antiguos sobre el tema, y ha servido como referencia obligada en la historia de la Educación en Arquitectura.

La formación académica en Arquitectura, se empieza a desarrollar a finales del siglo XVII. La École National Superior des Beaux Arts de Paris, constituida como el primer centro formal de enseñanza de la Arquitectura, caracterizada por sus fuertes vínculos formales y estilísticos con el pasado, recoge los logros de la Arquitectura clásica y el renacimiento en Europa; sirve como referencia para definir el papel del Arquitecto en la sociedad, diferenciándolo del constructor artesano, así como del ingeniero, convirtiéndose en líder, pensador y crítico de la Arquitectura y la Ciudad (Saldarriaga, 1996), hasta convertirse hoy día, como lo llama Umberto Eco, en "el último humanista de nuestro tiempo" (como se cita en Saldarriaga, 1996).

Los principios estéticos y conceptuales generados desde la Escuela de Bellas Artes de Paris, dominan la escena de la enseñanza de la Arquitectura, hasta que, en la segunda década del siglo XX, la necesidad de un cambio conceptual de la Arquitectura lleva a 
una redefinición de la enseñanza de la disciplina con efectos sobre el papel de la academia, y los intereses en el proceso de Enseñanza-Aprendizaje, En 1919 Walter Gropius da origen a una propuesta que congrega la Arquitectura, el Arte y la Tecnología, en lo que históricamente se ha denominado la escuela de la Bauhaus en Weimar, la cual plantea la mirada desde la Arquitectura y la formación en Arquitectura, sobre la necesidad de generar procesos industrializados tanto en la Construcción como en el Diseño, desde la reinterpretación de los logros de la Arquitectura clásica bajo una nueva mirada del desarrollo del lenguaje abstracto de la modernidad.

Luego de los cambios experimentados en la posguerra, cuando el propósito generalizado de la Arquitectura era atender de manera sistemática las necesidades de la gran demanda habitacional luego de la destrucción de la guerra, aparecen de manera aislada escuelas que desarrollan algunas de las ideas de industrialización de la Arquitectura que fueron planteadas por la Bauhaus, y lideran el cambio conceptual en la enseñanza de la Arquitectura hacia una internacionalización de saberes. La escuela de Philadelphia, la escuela de Comnel, el Instituto Técnico de Armour, actualmente Instituto Tecnológico de Illinois, que en su momento tuvo a la cabeza una de las grandes figuras de la Arquitectura como lo fue Mies Van der Rohe, quien, junto a otros grandes Arquitectos, influenciaron enormemente el panorama mundial de la Arquitectura, direccionando y moldeando el pensamiento de muchos Arquitectos en todos los rincones del mundo.

De manera paralela y como hecho atípico la "hermandad de Taliesin" de Frank Lloyd Wright, como hecho excepcional, aparece en el panorama de la historia de la enseñanza en Arquitectura, entendiendo la disciplina y su enseñanza, desde la formación integral del sujeto, desde una visión analítica y consciente del medio en el cual se emplaza, dando origen al pensamiento de una Arquitectura orgánica, en sintonía con la naturaleza.

A finales del siglo $X X$ y primera década del $X X I$, el panorama de la enseñanza de la Arquitectura, realmente no es tan diferente de la subdivisión de saberes básicos que son comunes a toda formación de un Arquitecto: diseño, construcción, historia y representación. A pesar de que es innegable observar la creciente reflexión por aspectos urbanos, tecnológicos y sobre todo la preocupación por generar respuestas mas amigables con el medio ambiente, las discusiones se centran en algunos aspectos formales de los procesos: las competencias, los créditos, la internacionalización, más que una reflexión profunda de las necesidades de formación en atención a las problemáticas locales.

A pesar de coexistir múltiples visiones que, de manera aislada, aportan a entender las diferentes problemáticas en la construcción social de la Arquitectura. Esta fase es marcada por la influencia de pequeñas elites, grupos y figuras de la Arquitectura promovidas por una revolución mediática del conocimiento, la Cooper Unión, los 5 de Nueva York, Rem Koolhaas, Frank Ghery, Zaha Hadid, entre otros aparecen en el panorama mundial, desarrollando conceptos de la Arquitectura que permean e influencian los procesos de enseñanza en muchas escuelas de Arquitectura alrededor del mundo; la cual es afectada ahora desde una visión desarrollista de una vanguardia formal y conceptual, que muchas veces no plantean acciones sobre problemáticas reales, y se traduce en una Arquitectura escenográfica de gran tendencia elitista y estratificada, contradictorias con la esencia del ser, desligada de una realidad, y de un mundo en crisis en búsqueda de su redefinición.

Es importante destacar como escenario de discusión y reflexión, el desarrollo de los Seminarios de Arquitectura Latinoamericana (SAL), los cuales, a partir de 1985, se han destacado como punto de encuentro y debate sobre el pensamiento arquitectónico latinoamericano, lo cual ha influido en la construcción de un lenguaje propio a partir de unos intereses comunes, que, en mayor o menor medida, es referente para la configuración de las escuelas de Arquitectura en Latinoamérica.

Al igual que los otros ámbitos educativos, la Educación en Arquitectura a comienzos del 2020, se adapta a las necesidades de dar continuidad a sus procesos académicos durante el aislamiento producto de la pandemia. Una formación que muy poco ha evolucionado a través de los años, se ha visto forzada a reinventarse en la Virtualidad, acomodando sus procesos a la nueva realidad.

\section{Arquitectura, Desarrollo y Educación: Cruce de Caminos y Puntos de Encuentro}

Desde las miradas que han sido planteadas con anterioridad, se pueden entrelazar los aspectos 
relativos al desarrollo social, los cuales, vistos desde un enfoque de la problemática ambiental, trascienden de manera directa a los temas educativos y a la Arquitectura. Es así como la influencia de la economía y toda la problemática planteada a través de la historia del desarrollo social de los seres humanos, no puede entenderse de manera aislada sino como una totalidad que envuelve tanto las acciones de los seres humanos, así como las decisiones que han afectado su formación, desarrollo, evolución y la manera de interactuar como especie en el planeta.

Es posible trazar como punto de partida las primeras líneas de conexión, para establecer el vínculo que permita entender la adopción de una postura de la academia, específicamente la que tiene que ver con la formación en Arquitectura, dada su importancia irrevocable como Arte y Ciencia integradora de saberes, y como uno de los aspectos más palpables del desarrollo del hombre en la historia.

En relación con el desarrollo sostenible que exige la problemática de la sociedad actual, es necesario repensar el papel del Educador. La enseñanza de la Arquitectura, como disciplina trasformadora, es imprescindible que sea repensada desde los cuestionamientos de los problemas de la realidad actual. La Arquitectura, como lo plantea Denk y Smajs (1999), es una de las partes más importantes de la cultura. La comprensión de la influencia de Arquitectura en el ambiente, es crucial para el entendimiento de la responsabilidad del ser humano hacia la problemática actual del desarrollo, tanto así que las formas en que evoluciona y se transforma la Arquitectura están conectadas con las afectaciones ecológicas y los problemas globales de la sociedad; de ahí la responsabilidad que debe asumir la academia en la formación de la Arquitectura, en establecer el punto de cambio para la conformación de una nueva "filosofía de la supervivencia" (Denk y Smajs, 1999), desde la Arquitectura y su relación cada vez más estrecha con el medio natural y la Ecología.

Si se revisa entonces el desarrollo de la Educación a través de la historia, se puede ver como esta se ha ido adaptando a los cambios que imponen las necesidades de la sociedad en las distintas épocas. Ante esta problemática de la crisis ambiental, social, y la nueva realidad post COVID-19, es necesario también repensar la forma como se han abordado los problemas desde la misma ciencia educativa, y es urgente disponer una actitud que asuma el reto de cambiar, en vez de adaptarse al cambio, ya que la crisis no da espera.

El reto, entonces, ante estas nuevas condiciones, es lograr una Educación que promueva una integración indisoluble entre el Hombre y la Naturaleza, desde una visión de la sostenibilidad de lo humano y no humano, una Educación integral, concebida esta como el entendimiento de las diferencias individuales, la formación del ser en integración y armonía con la naturaleza y la sociedad.

Es indudable que la formación integral en Arquitectura, requiere de algo más que superar el umbral de una especifica etapa universitaria de rígido esquema promocional. El Arquitecto integral denota una amplia configuración donde interviene la personalidad, el campo psicológico, proyectado adecuadamente en el medio social y cultural en su espacio-tiempo, la expresión cultural entendida dentro del marco intelectual, desde la cual incide la herencia, las conductas aprendidas y transmitidas por los miembros de una sociedad, y que son parte importante del bagaje con el cual los seres humanos enfrentamos el mundo.

El Ministerio de Educación incentiva a los Programas de Arquitectura del país a desarrollar curricularmente Planes de Estudio que logren reflejar factores identitarios que les den un valor agregado a sus Programas. Esta iniciativa bien podría fortalecer estas posturas ya planteadas sobre el fortalecimiento de la visión territorial de la Arquitectura. Los factores identitarios, definidos por el área de énfasis de cada Programa Académico, permite el desarrollo de reflexiones sobre los objetivos del, el perfil del ser humano que se pretende formar, y el impacto que se desea en el contexto (Martínez y Albis, 2018)

Este planteamiento, bien puede ser determinante en el carácter del profesional que se forma, de tal manera que logre desarrollar la capacidad de leer su contexto desde una postura crítica e integral y de esta forma conseguir una mayor sensibilidad al momento de abordar los problemas propios de la disciplina.

Este nuevo pensamiento de la formación en Arquitectura que debe gestionar la Universidad como espacio para la formación integral de los hombres, necesita pasar a otro estadio del desarrollo del pensamiento en el cual el tema principal gire en torno a las problemáticas del 
ambiente (López de Asiain, 2005), desde la visión de ser en relación armónica con la naturaleza, el estadio de la Noosfera o conjunto de seres inteligentes en armonía con el medio en que viven es el objetivo (Gómez, 2002).

$\mathrm{Si}$ se revisa el potencial de los países Latinoamericanos, sobre todo en el Caribe colombiano, dada sus condiciones geográficas y ambientales, las cuales pese a el retraso (Amar, 2002) y la crisis económica producto de las desigualdades sociales y económicas que plantea el modelo imperante, pueden jugar un papel importante en las transformaciones y transiciones que es necesario realizar e implementar para el desarrollo sostenible de la humanidad.

La formación en Arquitectura, es de vital importancia para jalonar fuertemente la conciencia para superar la crisis ecológica, debido a las capacidades de la Arquitectura y el diseño para delimitar formas de ser y habitar en el mundo (Fry, 1999; 2011; Escobar, 2016), el entendimiento del hábitat para la convivencia, la conservación de lo humano y no humano, entendido esto como una postura ética desde la Arquitectura, para la construcción de un mundo más equitativo.

Ante esta perspectiva problemática se pueden entrever una serie de interrogantes, por ejemplo: ¿Cómo lograr una Educación en Arquitectura, una academia que forme al Estudiante en la sensibilidad y cientificidad que le permita entender los problemas del territorio y lugar desde la conciencia del desarrollo sostenible? ¿Cómo generar una formación integral en Arquitectura, la cual esté enfocada hacia la conciencia y resolución de las problemáticas actuales de los grupos humanos? ¿Cómo generar un Currículo para la formación en Arquitectura, que atienda los problemas contemporáneos del habitar? ¿Cómo atender a los requerimientos que exige un mundo en crisis? ¿Qué reflexiones se deben propiciar ante las transiciones que se proponen al sistema mundo hegemónico? ¿Cuál es la Educación en Arquitectura que se necesita para afrontar las realidades de la nueva normalidad?

Estos y muchos otros interrogantes son los retos para la Arquitectura y los involucrados en estructurar su formación disciplinar, el entender estos requerimientos para enfrentar de una manera adecuada los retos que impone el nuevo milenio y la nueva normalidad post COVID-19, más aún la visión de la Educación en Arquitectura que urge desarrollar en los países latinoamericanos, sobre todo Colombia, por su posición estratégica y las condiciones ambientales exuberantes y favorables. Una nueva concepción pensada desde el sur y para el sur, con la capacidad de valorar las raíces culturales y a partir de ellas evolucionar hacia nuevos futuros posibles con equidad,

En el caso de Colombia las escuelas de Arquitectura aún tienen brechas importantes en el desarrollo de la Investigación, a pesar de que esta se encuentra transversalizada en los Currículos y en las Líneas de Investigación de los Grupos de Investigación. También es cierto que las redes académicas aún son débiles y los énfasis en los cuales se enfocan deben ser fortalecidos por equipos de académicos formados en las áreas ambientales y con el deseo de llevar la Universidad en el sentido de la sostenibilidad, es un gran avance en este sentido que actualmente, el $30 \%$ de los Programas de Arquitectura en el país desarrollan líneas de Investigación enfocadas en temas de Ciudad y Territorio, y otro $15 \%$ de las líneas de Investigación está enfocada en temas relacionados con Sostenibilidad, Medio Ambiente y Paisaje (Martínez y Albis, 2018).

La Arquitectura, como disciplina, enfrenta hoy una encrucijada en relación a las acciones a tomar en cuanto a la problemática del desarrollo desde la perspectiva ambiental; la academia, que históricamente ha respondido de manera importante a los requerimientos de la sociedad, en los últimos años se puede ver estancada bajo un liderazgo mediático de "falsas pretensiones" y figuras publicitarias. Es necesario cambiar la actitud de la academia frente a esta saturación formalista de alto impacto estético, y que representa las desigualdades en un mundo de desarrollo ambiguo.

La academia, como formadora de seres humanos conscientes, debe reflexionar sobre los problemas trascendentales de la Arquitectura para la Humanidad, cuya esencia es el dotar de refugio; la formación ligada a los aspectos economicistas de la explotación indiscriminada hace eco en la vanguardia tecnológica, el desarrollo per se, y la conformación de elites, olvidando que la Arquitectura es en esencia, una ocupación intelectual que se debe enfocar en la preservación de la vida y el planeta como el hábitat que une a todas las especies. 


\section{Conclusiones}

A manera de conclusiones, se presentan algunos puntos claves en el nuevo papel de liderazgo que la academia debe jugar en la formación integral de un Arquitecto, con el fin de entender y trascender los retos para la tercera década del siglo XXI.

La formación en Arquitectura requiere con urgencia una reflexión sobre la Formación Integral del ser y sobre la sostenibilidad de las acciones del Arquitecto hacia el futuro.

La Educación Integral del ser se logra trascendiendo la rutina de enseñar por enseñar, hacia el de la formación de un ser humano, entablando discusiones que atiendan entre otros: la Investigación en miras de la producción y acción, la conciencia ecológica, y la importancia del hábitat y la coexistencia de la multiculturalidad e interdisciplinariedad de saberes.

La Educación en Arquitectura, como proceso socializador, debe apuntar a la humanización desde una visión ética del actuar. El Arquitecto debe aportar al desarrollo social desde su capacidad integradora de saberes, y desde la sensibilidad que lo caracteriza y lo hace diferente. La Educación en Arquitectura se debe realizar desde lo situacional y lo local, en Latinoamérica esta visión genera todo un potencial innovador que conduciría a un desarrollo desde el aprovechamiento sustentable de la exuberancia del medio natural y cultural.

En el proceso de formación de un Arquitecto integral es necesario derribar las barreras disciplinares entre paisaje y Arquitectura, ciudad y territorio, en busca de una construcción de pensamiento que transforme el concepto de Arquitectura-Objeto, comprometido ahora con una idea de ArquitecturaHábitat-Ecología.

La Escuela de Arquitectura Latinoamericana esta llamada en este nuevo siglo a liderar las transiciones hacia un entorno amigable y sostenible, tanto para el ser humano como para todas las especies del planeta.

Desde esta visión, es pertinente la reflexión de Arciniegas (1937) en relación con el cambio actitudinal del hombre para enfrentar los problemas futuros, cuando explica que uno de los mayores logros de los humanos será la comprensión de las simples leyes naturales y actuar en consecuencia; llegar a ese conocimiento de forma humilde, sin imponer la propia voluntad.

\section{REFERENCIAS}

Amar, J. J. (2002). La región caribe y la hipótesis del retraso cultural. Investigación y desarrollo Vol. 11 (1), 2-25.

Arciniégas, G. (1937). América tierra firme: sociología. Santiago de Chile: Ediciones Ercilla.

Ballesteros, J. y Pérez Adán, J. (1997, eds.). Sociedad y Medio Ambiente. Madrid, España: Editorial Trotta S.A.

Climate Action Summit (2019). Report of the secretary-general on the 2019 climate action summit and the way forward in 2020. Recuperado de: https://www.un.org/en/climatechange/assets/pdf/cas report 11 dec.pdf

Denk, Z., Smajs, J. (1999). Reconstrucción Bio - amistosa de las ciudades. Memorias XX congreso Unión internacional de Arquitectos, Volumen 2. Beijing, China: UIA.

DNP (2019). Objetivos de desarrollo sostenible. La agenda 2030 en Colombia. Recuperado de: https://www. ods.gov.co/es

Escobar, A. (2016). Autonomía y diseño: La realización de lo comunal. Popayán: Universidad del Cauca. Sello Editorial.

Fry, T. (1999). A new design philosophy: an introduction to defuturing. Sydney: UNSW Press.

Fry, T. (2011). Design as Politics. Oxford, New York: Berg. 
FAO (1996). Enseñanzas de la revolución verde: hacia una nueva revolución verde. Documentos técnicos de referencia. Recuperado de: http://www.fao.org/3/w2612S/w2612s06.htm

Gómez, L. J. (2002). Biosfera, Noosfera y Gaia: tres conceptos para reflexionar sobre el desarrollo sostenible. Revista de Extensión Cultural (46): 14- 20. Recuperado de: http://www.bdigital.unal.edu.co/53910/1/ luisjairgomezgiraldo.2002.pdf

livari, N., Sharma, S., y Venta-Olkkonen, L. (2020). Digital transformation of everyday life -How COVD-19 pandemic transformed the basic education of the young generation and why information management research should care? International Journal of information management. Article in press. Recuperado de: https://www.sciencedirect.com/science/article/pii/S0268401220310264?via\%3Dihub

Leff, E. (2000). Perspectiva ambiental del desarrollo del conocimiento, los problemas del conocimiento y la perspectiva ambiental del desarrollo. México: Ed. Siglo XXI.

Lokhandwala, S., y Gautam, P. (2020). Indirect impact of COVID-19 on environment: a brief study in Indian context. Environmental Research 188. Article in press. Recuperado de: https://www.sciencedirect. com/science/article/pii/S0013935120307027?via\%3Dihub

López de Asiain, María. (2005). La formación Medio Ambiental del Arquitecto. Tesis Doctoral, Escuela de Arquitectura de Barcelona, octubre de 2005. Departamento de construcciones arquitectónicas de la Universidad politécnica de Cataluña. Recuperado de: https://pdfs.semanticscholar.org/7ca5/ cda56fbf53817d524a3d6799e04718e35af1.pdf

Madurai, R., y Pugazhendhi, R. (2020). Restructured society and environment: a review on potential tecnological strategies to control the COVID-19 pandemic. Science of the Total Environment 724. Article in press. Recuperado de: https://www.sciencedirect.com/science/article/pii/ S0048969720323755?via\%3Dihub

Martínez, G. y Albis, M.C. (2018, Comp.). Enfoques, Teorías y Perspectivas de la Arquitectura y sus Programas Académicos. Sincelejo: CECAR. Recuperado de: https://cecar.edu.co/documentos/editorial/e-book/ enfoques-teorias-y-perspectivas-de-la-Arquitectura-y-sus-Programas-academicos.pdf

Red de transición (2019). El movimiento de Transición en el Estado español. Actualización del mapa de iniciativas locales de Transición (2014-2017). Recuperado de: http://www.reddetransicion.org/wpcontent/uploads/2020/02/Informe-El-Movimiento-de-Transicion-en-el-Estado-espa\%C3\%B1olResumen-cast.pdf

Sistig, B. (2018, Ed.). A decade of positive visión and practical action. Grey Lynn 2030. New Zealand: Grey Lynn Transition community. Recuperado de: https://transitionnetwork.org/wp-content/uploads/2019/02/ GL2030-book-text-final-s-Oct-2018.pdf

Saldarriaga Roa, A. (1996). Aprender Arquitectura, un manual de supervivencia, Ed. Corona S.A, Bogotá, 1996.

Somani, M., Srivastava, A.N., Kumar, S., y Sharma, A. (2020). Indirect implications of COVID-19 towards sustainable environment: an investigation in Indian context. Bioresource Technology Reports 11. Article in press. Recuperado de: https://www.sciencedirect.com/science/article/pii/ $\underline{\text { S2589014X20301122?via\%3Dihub }}$

Tuning- América Latina (2007). Reflexiones y perspectivas de la Educación superior en América Latina. Informe final - Proyecto Tuning- América Latina 2004 - 2007. Bilbao: Publicaciones de la Universidad de Deusto. Recuperado de: http://tuningacademy.org/wp-content/uploads/2014/02/TuningLAlll Final-Report SP.pdf

Wijesooriya, N., Mishra, V., Brand, P., y Rubin, B. (2020). COVID-19 and telehealth, education, and research adaptations. Paediatric Respiratory Reviews. Article in press. Recuperado de: https://www. sciencedirect.com/science/article/pii/S1526054220300944?via\%3Dihub 